\title{
Low Complexity Frequency-Domain Despreading for Cyclic-Prefix CDMA Systems
}

\author{
Pin-Hsun Lin, Student Member, IEEE, and Tzi-Dar Chiueh, Senior Member, IEEE
}

\begin{abstract}
In this letter, a frequency-domain despreading method for the cyclic-prefix code-division multiple-access (CP-CDMA) system is introduced. Using the orthogonality transformation property of the discrete Fourier transform (DFT), we can despread the received CP-CDMA signals in the frequency domain. Moreover, we propose an efficient architecture for the proposed frequency-domain despreader. Comparison with the conventional time-domain despreading approach shows that the proposed architecture can save a large amount of computation. The proposed scheme is therefore suitable for efficient implementation of CP-CDMA receivers that adopt frequency-domain equalization and despreading.
\end{abstract}

Index Terms-Code division multiple access (CDMA), cyclic-prefix, frequency-domain despreading, fast Fourier transform (FFT).

\section{INTRODUCTION}

C ONVENTIONAL direct-sequence code-division multiple access (DS-CDMA) receivers use RAKE combining to collect the signal components dispersed in time when the transmitted signal passes through the multipath fading channel [1]. In practice, the number of fingers in a RAKE combiner is limited due to the constraint in hardware complexity. Such limited number of RAKE fingers degrades the receiver performance in those cases when the multipath fading is severe.

Recently, a frequency-domain equalization (FEQ) technique for receiving single-carrier signals was proposed [2]. The main advantage of FEQ lies in its low complexity when compared with the time-domain equalizer and the RAKE combiner. In [3], a receiver utilizing the FEQ technique was proposed for receiving DS-CDMA signals augmented by cyclic prefix, called cyclic-prefix CDMA (CP-CDMA). It was also shown that a receiver with FEQ can receive the CP-CDMA signal much better than a RAKE receiver in receiving DS-CDMA signal, especially when the system loading is high. The concept of frequency-domain processing for DS-CDMA signals can also be found in [4], where multiuser detection is achieved in the frequency domain. In another work, the CP-CDMA receiver with FEQ is shown to perform comparably with the multi-carrier CDMA (MC-CDMA) system [5]. The MC-CDMA system performs both equalization and despreading in the frequency domain while the conventional CP-CDMA system performs

Manuscript received August 12, 2003. The associate editor coordinating the review of this letter and approving it for publication was Prof. A. Annamalai. This work was supported by National Science Council, Taiwan, R.O.C., under Grant NSC92-2219-E-002-012 and NSC92-2220-002-002.

The authors are with the Department of Electrical Engineering, National Taiwan University, Taipei 10617, Taiwan, R.O.C. (e-mail chiueh@cc.ee.ntu.edu.tw).

Digital Object Identifier 10.1109/LCOMM.2004.828176 equalization in the frequency domain and despreading in the time domain. The comparison indicates that the CP-CDMA system has only slightly higher bit error rate (BER) than the MC-CDMA system. A unified framework of single- and multi-carrier CDMA systems based on the FEQ technique is discussed in [6], where demodulation of both CDMA and multi-carrier-modulation (MCM) signals within a single receiver is shown possible.

In this letter we focus on the issue of despreading the equalized CP-CDMA signal in the frequency domain and a low-complexity architecture for such operation. In Section II, the CP-CDMA system model will be introduced. We also illustrate how to perform frequency-domain despreading in a CP-CDMA receiver. In Section III, an efficient architecture for frequency-domain despreading, derived from the inverse fast Fourier transform (IFFT) architecture, is proposed. By exploiting the relationship between frequency-domain codewords for despreading, the new architecture saves much complexity. Finally, a conclusion is given in Section IV.

\section{SySTEM MODEL}

A downlink CP-CDMA system model is shown in Fig. 1. Let $s_{m}(i)$ and $\boldsymbol{c}_{m}$ denote the $i$ th data symbol and the spreading code for user $m$, respectively. The spreading code, $\boldsymbol{c}_{m}$, is an $L \times 1$ column vector, i.e., the codes are $L$-chip long. Without loss of generality, let $m=0$ denote the pilot signal for channel estimation and there are $M$ active users, so $0 \leq m \leq M$ and $M+1 \leq L$ since both the pilot code and the user codes are Walsh codes with length $L$. After collecting $2^{k}$ consecutive symbols from all $M$ users and the pilot, we have

$$
\boldsymbol{s}=\left[\sum_{m=0}^{M} s_{m}(0) \boldsymbol{c}_{m}^{T} \ldots \sum_{m=0}^{M} s_{m}\left(2^{k}-1\right) \boldsymbol{c}_{m}^{T}\right]^{T}
$$

where $s$ is an $N \times 1$ vector and $N=2^{k} L$. A block is constructed by adding a cyclic prefix to $s$. Note that the length of the cyclic prefix is made longer than the maximum excess delay of the channel to accommodate the inter-symbol interference (ISI).

At the receiver, the received block after removal of cyclic prefix can be formulated as

$$
\boldsymbol{r}=\boldsymbol{H} \boldsymbol{s}+\boldsymbol{n}
$$

where $\boldsymbol{H}$ is the $N \times N$ circulant matrix formed by the channelimpulse response and $\boldsymbol{n}$ is the Gaussian noise vector. Taking the discrete Fourier transform (DFT) of the received block, we have

$$
W r=W H s+\nu=G W s+\nu
$$




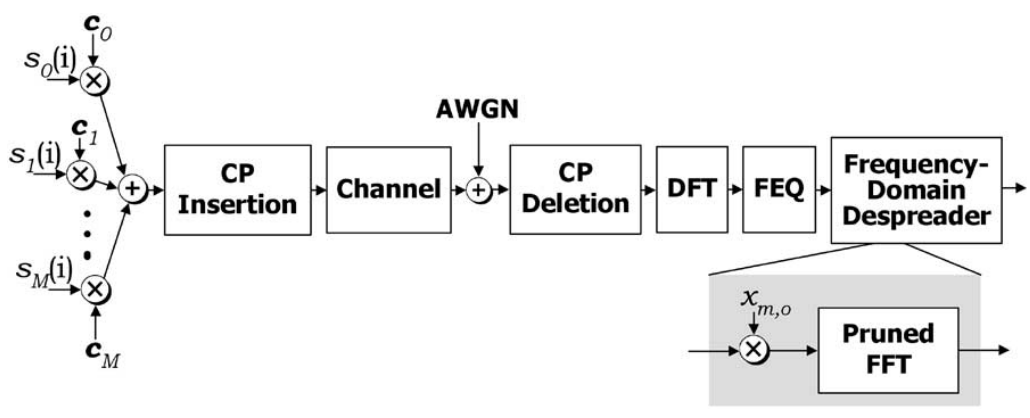

Fig. 1. The downlink frequency-domain despreading CP-CDMA system model.

where $\boldsymbol{W}$ is the $N \times N$ normalized DFT matrix, i.e., $\boldsymbol{W} \boldsymbol{W}^{H}=$ $\boldsymbol{I} ; \boldsymbol{\nu}$ is the frequency-domain noise vector; and $\boldsymbol{G}$ is the $N \times N$ diagonal matrix whose diagonal terms correspond to the DFT of the first row of $\boldsymbol{H}$ and $\boldsymbol{G}=\boldsymbol{W} \boldsymbol{H} \boldsymbol{W}^{H}$. Several FEQ algorithms mentioned in [6] can be used to accomplish equalization of the received frequency-domain signal. Here we assume the effect of the channel impulse response can be eliminated for simplicity and the equalized signal is given by $\boldsymbol{W} \boldsymbol{s}^{\prime}$, where $\boldsymbol{s}^{\prime}$ is the transmitted signal with noise, possibly enhanced by FEQ. In receivers that use time-domain despreading, the desired user's signal can then be retrieved by an inverse DFT, followed by a time-domain despreading operation.

In fact, the inverse DFT followed by the time-domain despreading can be shown to be mathematically equivalent to frequency-domain despreading. Take the W-CDMA system as an example, $N=2^{k} L=256, k=0,1, \ldots, 6$. In this case, the CP-CDMA transmitter concatenates $2^{k} L$-chip data symbols to form a block, which is then prefixed with the cyclic prefix. The conventional approach of despreading the signal in the time domain using the $m$ th user's code on the $i$ th symbol is

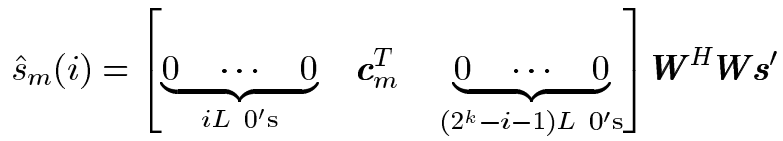

$$
\begin{aligned}
& =\boldsymbol{x}_{m, i}^{T} \boldsymbol{W} \boldsymbol{s}^{\prime}
\end{aligned}
$$

where $\hat{s}_{m}(i)$ is the estimated $i$ th symbol of the $m$ th user and $\boldsymbol{x}_{m, i}$ is the frequency-domain spreading code. The received signal can be despread in the frequency domain and the inverse DFT operation can be saved. Moreover, note that

$$
\boldsymbol{x}_{m, i}^{H} \boldsymbol{x}_{n, j}=\boldsymbol{c}_{m}^{T} \boldsymbol{c}_{n} \delta_{i, j}=L \delta_{m, n} \delta_{i, j} .
$$

\section{LOW COMPLEXITY FREQUENCY-DOMAIN DESPREADING}

Although $\boldsymbol{x}_{m, i}$ can be used to despread the $i$ th data symbol of user $m$, the computational complexity can be too high. Since $2^{k}$ frequency-domain despreading operations are required to estimate all symbols in the block. However, note that the time-domain spreading codes of a particular user are circular-shifted versions of one another. Consequently, the frequency-domain spreading codes can be reformulated as

$$
\begin{aligned}
\boldsymbol{x}_{m, i}^{T} & =\left[\begin{array}{llll}
e^{j 0} & e^{\frac{j 2 \pi(i L)}{N}} & e^{\frac{j 2 \pi(2 i L)}{N}} \cdots & e^{\frac{j 2 \pi(N-1)(i L)}{N}}
\end{array}\right] \boldsymbol{X}_{m} \\
& =\boldsymbol{w}_{(i L)} \boldsymbol{X}_{m}
\end{aligned}
$$

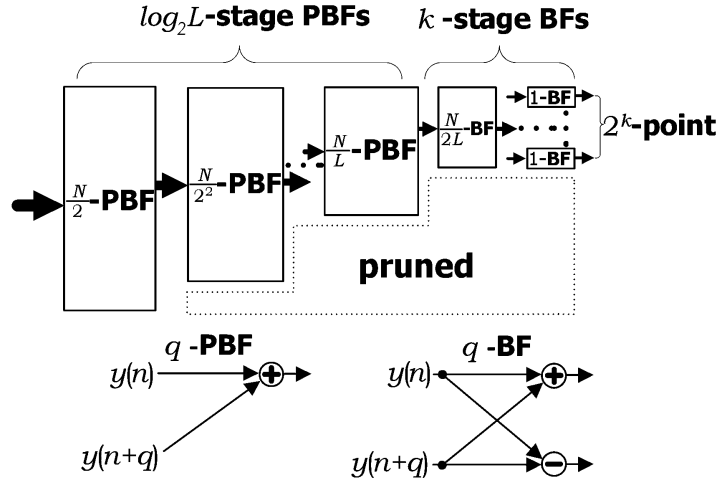

Fig. 2. A pruned IFFT architecture used in the proposed frequency-domain despreader.

where $\boldsymbol{X}_{m}$ is the diagonal matrix whose diagonal entries are the elements in $\boldsymbol{x}_{m, 0}$, and $\boldsymbol{w}_{(i L)}$ is the $(i L)$ th row of $\boldsymbol{W}^{H}$.

Replacing (3) into (2) and stacking $2^{k}$ equations on top of each other, we have

$$
\begin{aligned}
\left(\begin{array}{c}
\hat{s}_{m}(0) \\
\hat{s}_{m}(1) \\
\vdots \\
\hat{s}_{m}\left(2^{k}-1\right)
\end{array}\right) & =\left(\begin{array}{c}
\boldsymbol{w}_{0} \\
\boldsymbol{w}_{L} \\
\vdots \\
\boldsymbol{w}_{\left(2^{k}-1\right) L}
\end{array}\right) \boldsymbol{X}_{m} \boldsymbol{W} \boldsymbol{s}^{\prime} \\
& =\left.\boldsymbol{W}^{H} \boldsymbol{X}_{m} \boldsymbol{W} \boldsymbol{s}^{\prime}\right|_{\downarrow_{L}} .
\end{aligned}
$$

Equation (4) indicates that in order to retrieve all $2^{k}$ symbols of user $m$, the received block is first transformed to the frequency domain, equalized, and component-wise multiplied by the primary frequency-domain code $\left(\boldsymbol{x}_{m, 0}\right)$, transformed back to the time domain, and decimated.

It is well known that the DFT operation can be efficiently implemented by the FFT structure. Moreover, the decimateby- $L$ operation makes it sufficient to compute only part of the inverse DFT operation. Taking the radix-2 decimation-in-frequency IFFT as an example, the $2^{k}$ decimated-by- $L$ time-domain signals are actually the first $2^{k}$ signals in the IFFT outputs. This is because the $2^{k}$ decimated time-domain signals all have indices that end with $\log _{2} L$ zeros and these indices, when bit reversed, become the first $2^{k}$ indices with $\log _{2} L$ leading zero bits. Thus the top $2^{k}$ IFFT outputs (in bit-reversed order) are the decimated-by- $L$ time-domain signals and the remaining $N-2^{k}$ IFFT outputs are useless. Therefore, we can prune the IFFT architecture and obtain an architecture for the frequency-domain despreading scheme, as shown in Fig. 2. 
TABLE I

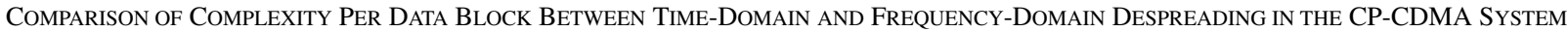

\begin{tabular}{c||c|c||c|c||c|c}
\hline \multicolumn{1}{c||}{} & \multicolumn{2}{c||}{$\begin{array}{c}\text { Time-Domain } \\
\text { Despreading }\end{array}$} & $\begin{array}{c}\text { Frequency-Domain } \\
\text { Despreading }\end{array}$ & \multicolumn{2}{c}{$\begin{array}{c}\text { Complexity } \\
\text { Saving }\end{array}$} \\
\hline $\begin{array}{c}\text { Spreading } \\
\text { Factor }\end{array}$ & $\begin{array}{c}\text { CM } \\
(\#)\end{array}$ & $\begin{array}{c}\text { CA } \\
(\#)\end{array}$ & $\begin{array}{c}\text { CM } \\
(\#)\end{array}$ & $\begin{array}{c}\text { CA } \\
(\#)\end{array}$ & $\begin{array}{c}\text { CM } \\
(\%)\end{array}$ & $\begin{array}{c}\text { CA } \\
(\%)\end{array}$ \\
\hline$L=256$ & 768 & 2303 & 256 & 255 & 66.67 & 88.93 \\
\hline$L=128$ & 768 & 2302 & 256 & 256 & 66.67 & 88.88 \\
\hline$L=64$ & 768 & 2300 & 257 & 260 & 66.54 & 88.70 \\
\hline$L=32$ & 768 & 2296 & 261 & 272 & 66.02 & 88.15 \\
\hline$L=16$ & 768 & 2288 & 273 & 304 & 64.45 & 86.71 \\
\hline$L=8$ & 768 & 2272 & 305 & 384 & 60.29 & 83.10 \\
\hline$L=4$ & 768 & 2240 & 385 & 576 & 49.87 & 74.26 \\
\hline
\end{tabular}

Those operations enclosed by dotted lines denote redundant computation that can be saved. The remaining butterfly (BF) computations can be further divided into two types: $\log _{2} L$ stages starting from the input use the pruned $\mathrm{BF}(\mathrm{PBF})$ and the following $\log _{2}(N / L)=k$ stages use the conventional BF. Note that the complexity of the PBF is only half that of the BF. Since the number of complex twiddle-factor multiplication (CM) in the bottom part of the FFT signal flow graph are more than that in the top part, another advantage of such lower triangular FFT pruning is more saving in twiddle-factor multiplication.

The number of complex multiplications (CM) and the number of complex additions (CA) needed per despread data block of the proposed frequency-domain spreading scheme and those of the conventional time-domain despreading using IFFT [3] are listed in Table I. The IFFT size $(N)$ is 256 and the spreading factor $(L)$ is from 4 to $256(k=6,5, \ldots, 0)$. From this table we can see that a large amount of complexity is saved even when $L$ is as small as 4 .

\section{CONCLUSION}

In this letter, we propose a low-complexity frequency-domain despreading scheme for the CP-CDMA receivers. The received signal can be despread in the frequency domain using codes that have been transformed to the frequency domain in advance. When the CP is longer than a CDMA symbol, we can concatenate several symbols as a block before inserting the $\mathrm{CP}$. In this case, using a pruned IFFT architecture we can still accomplish the frequency-domain despreading efficiently. Comparison in computation complexity shows the proposed scheme outperforms the original CP-CDMA system using time-domain despreading. The proposed scheme thus opens a new way of demodulating the CP-CDMA signal in the frequency domain and also makes it possible to construct a dual-mode frequencydomain receiver that can demodulate both CDMA-based and MCM-based signals.

\section{REFERENCES}

[1] M. K. Simon, J. K. Omura, R. A. Scholtz, and B. K. Levitt, Spread Spectrum Communications Handbook. New York: McGraw-Hill, 1994.

[2] D. Falconer, S. L. Ariyavisitakul, A. Benyamin-Seeyar, and B. Eidson, "Frequency domain equalization for single-carrier broadband wireless systems," IEEE Commun. Mag., vol. 40, no. 4, pp. 58-66, Apr. 2002.

[3] K. L. Baum, T. A. Thomas, F. W. Vook, and V. Nangia, "Cyclic-prefix CDMA: An improved transmission method for broadband DS-CDMA cellular systems," in Wireless Communications and Networking Conf., vol. 1, Mar. 2002, pp. 183-188.

[4] C. M. Chang and K. C. Chen, "Frequency-domain approach to multiuser detection in DS-CDMA communications," IEEE Commun. Lett., vol. 4, pp. 331-333, Nov. 2000.

[5] F. Adachi, T. Sao, and T. Itagaki, "Performance of multicode DS-CDMA using frequency domain equalization in frequency selective fading channel," Electron. Lett., vol. 39, no. 2, pp. 239-241, Jan. 2003.

[6] L. Bruhl and B. Rembold, "Unified spatio-temporal frequency domain equalization for multi- and single-carrier CDMA systems," in Proc. IEEE Vehicular Technology Conf., vol. 2, Sept. 2002, pp. 24-28. 